\title{
ANOMALIIAS CONGÉNITAS DE LOS EMBERA-CHAMÍ EN LOS RESGUARDOS INDÍGENAS DE RIOSUCIO, CALDAS, COLOMBIA, DE 2007 A 2012
}

Recibido: junio del 2018

Aceptado: marzo del 2019

Eduardo Lozano ${ }^{1}$, Lorena Vargas ${ }^{2}$

\section{Resumen}

Introducción: Tal como sucede a nivel mundial, en Colombia las malformaciones congénitas son causas importantes de mortalidad infantil, enfermedad crónica y discapacidad. Las poblaciones indígenas de Colombia no cuentan con estudios definidos a este respecto, por lo cual la presente investigación se desarrolla en la zona indígena. Objetivo general: Caracterizar las anomalías congénitas de los indígenas EmberaChamí residentes en los resguardos del municipio de Riosucio, Caldas, entre los años 2007 y 2012. Métodos: Estudio descriptivo, retrospectivo, aplicado a 125 nacidos vivos con anomalías congénitas. Se aplicó el formato especializado de Instituto Nacional de Salud (INS) de Colombia y para el análisis de variables se utilizó el paquete estadístico SPSS. Resultados: De 3.382 nacimientos el 3,7\% (125) presentó anomalías congénitas. Se encuentra una asociación entre la edad de la madre y la presencia de anomalías congénitas. En el 87,2\% de los casos fueron con edad gestacional de término. Las anomalías más frecuentes fueron: displasia de cadera, criptorquidia, malformaciones cardiovasculares, pie equino e hidrocefalia. Conclusiones: Se encuentra una incidencia de anomalías congénitas en la población indígena de los Embera-Chamí de Riosucio, Caldas, mayor que para el promedio de la población general en Colombia.

Palabras clave: anomalía congénita, incidencia, población indígena.

${ }^{1}$ Medico epidemiólogo, Epidemiólogo de Campo.

${ }^{2}$ Enfermera jefe y directora de la Secretaría de Salud de Riosucio, Caldas. 


\section{CONGENITAL MALFORMATIONS IN EMBERA-CHAMI IN INDIGENOUS RESERVATIONS IN RIOSUCIO, CALDAS, COLOMBIA, FROM 2007 TO 2012}

Eduardo Lozano ${ }^{1}$, Lorena Vargas ${ }^{2}$

\section{Abstract}

Introduction: As it happens worldwide, in Colombia, congenital malformations are important causes of child mortality, chronic disease and disabilities. Given that indigenous populations in Colombia do not count with specific research about this topic, this work is developed in an indigenous area. Objective: To characterize congenital abnormalities in Embera-Chamí indigenous people who live in the reservations of Riosucio, Caldas, between 2007 and 2012. Method: Descriptive and retrospective study applied on 125 newborns who had congenital malformations. We used the specialized format from Colombia's National Health Institute and we used SPSS to analyze the variables. Results: Out of 3.382 births, a $3.7 \%$ (125) had congenital malformations. We associated the age of the mother with the presence of said malformations. $87.2 \%$ of cases had term gestational age. Most frequent malformations were: hip dysplasia, cryptorchidism, cardiovascular malformations, clubfoot and hydrocephaly. Conclusions: There is a higher incidence of congenital malformations in the Embera-Chamí people, compared to the average of general population in Colombia. 
Eduardo Lozano ${ }^{1}$, Lorena Vargas ${ }^{2}$

\section{Resumo}

Introdução: assim como acontece em outros países, na Colômbia, as más-formações congênitas são causas importantes de mortalidade infantil, doença crônica e deficiência. As populações indígenas colombianas não contam com estudos definidos sobre isso, portanto esta pesquisa é desenvolvida na zona indígena. Objetivo geral: caracterizar as anomalias congênitas dos indígenas Embera-Chamí residentes nas reservas do município de Riosucio, Caldas, entre 2007 e 2012. Métodos: estudo descritivo, retrospectivo, aplicado a 125 nascidos vivos com anomalias congênitas. Foi aplicado o formulário especializado do Instituto Nacional de Saúde da Colômbia; para a análise de variáveis, foi utilizado o pacote estatístico SPSS. Resultados: de 3.382 nascimentos, 3,7 \% (125) apresentaram anomalias congênitas. Verifica-se uma associação entre a idade da mãe e a presença de anomalias congênitas. Em 87,2 \% dos casos foram com idade gestacional de termo. As anomalias mais frequentes foram: displasia do quadril, criptorquidia, más-formações cardiovasculares, pé equino e hidrocefalia. Conclusões: constata-se uma incidência de anomalias congênitas na população indígena dos Embera-Chamí de Riosucio, Caldas, maior que para a média da população geral na Colômbia. 


\section{Introducción}

Este estudio se desarrolló en Colombia en el departamento de Caldas, municipio de Riosucio, el cual está localizado a dos horas al occidente de Manizales, la capital de Caldas, donde el $80 \%$ de la población es indígena de una línea étnica llamada Embera-Chamí (1-3).

En Colombia, los resguardos indígenas son propiedad colectiva de sus habitantes nativos y son inalienables, imprescriptibles e irrevocables. Los resguardos indígenas son formados por uno o más grupos indígenas, son una unidad territorial, étnica, política, económica y jurídica. Sus habitantes son dueños de la tierra para su manejo y son gobernados por una organización autónoma llamada gobernación indígena, con sus lineamientos propios y tradiciones culturales (4-9).

Esta comunidad indígena que tiene más de 600 años de historia y aproximadamente una población de 50000 personas, quienes sobrevivieron a la colonización española y continuaron con la intención de preservar sus propias tradiciones y hábitos culturales (10).

Riosucio, Caldas, está dividido en cuatro resguardos indígenas llamados: La Montaña, Cañamomo-Lomaprieta, Escopetera-Pirsa y San Lorenzo. Se identifican en el municipio la zona urbana y la rural, la cual incluye dos corregimientos: San Lorenzo y Bonafont. El resguardo de San Lorenzo tiene una población de 11217 personas, La Montaña 18292 habitantes, Escopetera-Pirsa 7.856 indi-

viduos y Cañamomo-Lomaprieta 22391 habitantes (11-12).
Los Embera-Chami han mantenido costumbres nativas y endogamia, lo cual puede haber aumentado el riesgo de anomalías congénitas en sus comunidades (13-17). El $94 \%$ de los niños nacen en el hospital público local, lo que implica una pequeña cantidad de recién nacidos no registrados. Tanto el gobierno como las autoridades indígenas solicitan un registro de nacimiento, pero las historias clínicas se inician solo hasta la primera visita del niño al sistema general de salud de la nación. $\mathrm{Al}$ existir como parte de su cultura un concepto de salud propio, esto implica que los recién nacidos pueden ser manejados por sus propias parteras $y$ médicos tradicionales y no se tiene una historia clínica en el sistema de salud oficial colombiano de estos menores (18-21).

Colombia tiene un sistema de vigilancia epidemiológica de anomalías congénitas para su población en general (22-24); sin embargo, a pesar de la cantidad de población indígena, la caracterización de anomalías congénitas en los habitantes y grupos indígenas no se ha hecho y es importante identificar la carga de enfermedades de este tipo en estas microculturas, por lo cual surge el propósito de este estudio que se hizo descriptivo y retrospectivo.

\section{Materiales y métodos}

El objetivo general propuesto fue caracterizar las anomalías congénitas que se presentaron en la comunidad indígena Embera-Chamí, residente en los resguardos del municipio de Riosucio, Caldas, entre el 2007 y el 2012. Se buscó 
comparar la tasa de incidencia de malformaciones congénitas entre la población indígena Embera-Chamí de Riosucio, Caldas, con la población general en Colombia.

Con el fin de obtener la aprobación para el desarrollo de la investigación por parte de los cabildantes de la comunidad Embera-Chamí, se llevaron a cabo dos eventos de socialización previos al inicio del estudio: se desarrolló un estudio descriptivo y retrospectivo, inicialmente se identificó a los niños nacidos con anomalías congénitas en el municipio de Riosucio entre 2007 y 2012, mediante los registros del Sivigila (Sistema de Vigilancia de Salud Pública) que identifica los casos con malformaciones congénitas, luego se acudió a las historias clínicas de estos casos, para lo cual se utilizaron los registros de "nacidos vivos" del Hospital San Juan de Dios de Riosucio, con lo que se logró establecer la procedencia por resguardo indígena.

Para la recolección de datos se utilizó el formato que el Instituto Nacional de Salud de Colombia desarrolló e incluyó en los protocolos de seguimiento a las anomalías congénitas (25-30). Se aplicó la definición de malformaciones congénitas o defectos congénitos que el instrumento contempla, la cual es: anormalidades estructurales o funcionales de los órganos, sistemas o partes del cuerpo que se producen durante la vida intrauterina (25).

Se entrenaron unos recolectores de información que aplicaron el instrumento en hospitales e instituciones de salud en Riosucio y Manizales. Las principa- les fuentes de información fueron las historias clínicas del Hospital San Juan de Dios de Riosucio, institución de segundo nivel y de entidades de alta complejidad de Manizales, como el Hospital Infantil de la Cruz Roja, la Fundación Pequeño Corazón y Medintegral, donde los pacientes fueron evaluados porque presentaron algún tipo de anomalía congénita identificada en los primeros seis meses de edad de los nacidos entre 2007 y 2012.

Del total de los casos registrados como nacidos vivos en el municipio de Riosucio, Caldas, en el periodo 2007-2012, se utilizó el instrumento "Anomalías Congénitas" Cód. INS: 735 del Instituto Nacional de Salud, para la clasificación de estas patologías. Inicialmente se reportaron 600 casos de pacientes a quienes se les identificaron anomalías congénitas, se excluyeron casos de infecciones prenatales y de bajo peso al nacer con malformaciones, que los genetistas no consideran incluidos en malformaciones congénitas (26), pero que estaban incluidos en el instrumento del Instituto Nacional de Salud de Colombia.

Los criterios de inclusión fueron los siguientes:

1) Historias clínicas que correspondieran a madres indígenas, confirmadas dentro de los listados censales de los resguardos indígenas, procedentes del municipio de Riosucio, Caldas, Colombia.

2) Menores de cinco años nacidos de las madres de las historias clínicas 
del criterio 1, con malformaciones congénitas o anomalías incluidas en el protocolo del Instituto Nacional de Salud de Colombia de malformaciones y los criterios de exclusión fueron: recién nacidos con bajo peso al nacer, fimosis y malformaciones causadas por infecciones presentadas durante el embarazo (31-33). Luego de que se aplicaron estos criterios se determinaron 125 casos en la investigación, al confirmar el diagnóstico de malformación y su procedencia.

Se realizó un análisis estadístico descriptivo de los hallazgos en los 125 casos de malformaciones congénitas de los pacientes indígenas nacidos en Riosucio, entre 2007 y 2012. Las variables incluidas fueron: año de nacimiento, edad de primera consulta médica, sexo, resguardo indígena en donde nació el niño, procedencia, sitio de residencia, edad de la madre, edad gestacional al nacimiento, diagnóstico principal, sistema fisiológico comprometido y diagnóstico secundario. Las variables mencionadas fueron cruzadas utilizando medidas de asociación en tablas $2 \times 2$ (factor de riesgo versus enfermedad o daño), se aplicó el ji cuadrado y se consideró estadísticamente significativo el $\mathrm{p} \leq 0,05$. El resultado de ji cuadrado se aplica para contrastar frecuencias observadas con las frecuen- cias esperadas. Se utilizó el programa Excel para recolectar la información y esta fue procesada en el paquete estadístico SPSS versión 19.

\section{Consideraciones éticas}

De acuerdo a los estándares científicos, técnicos y administrativos para las investigaciones en salud establecidas en la Resolución 008430 de 1993 del Ministerio de Salud de Colombia (34), esta investigación no presenta ningún riesgo $y$ las instituciones participantes aceptaron el consentimiento informado por escrito, en el cual se especificó la confidencialidad de la información recolectada de los pacientes.

\section{Resultados}

Durante el periodo de la investigación hubo 3382 nacimientos en los resguardos indígenas, de acuerdo al censo indígena local. De estos, 125 casos (3,7\%) presentaron alguna anomalía congénita. De los 125 casos, 63 casos $(50,4 \%)$ fueron de sexo femenino y 62 casos $(49,6 \%)$ fueron de sexo masculino. La frecuencia de casos encontrados, entre 2007 y 2012 fue similar (tabla 1), aunque en la tasa de anomalías congénita entre 2009 y 2011 se nota un aumento (gráfico 1). 
Tabla 1. Tasas de anomalía congénita en la comunidad indígena de Riosucio, entre los años 2007 y 2012, basados en el censo indígena local por cada 1.000 nacidos vivos

\begin{tabular}{l|c|c|c|c|c|c}
\hline \multicolumn{1}{c|}{ Años } & $\mathbf{2 0 0 7}$ & $\mathbf{2 0 0 8}$ & $\mathbf{2 0 0 9}$ & $\mathbf{2 0 1 0}$ & $\mathbf{2 0 1 1}$ & $\mathbf{2 0 1 2}$ \\
\hline Nacidos vivos & 608 & 591 & 472 & 645 & 647 & 419 \\
Malformaciones congénitas & 20 & 17 & 24 & 22 & 28 & 14 \\
Tasa $\times 1.000$ nacidos vivos & 32,8 & 28,7 & 50,9 & 34,1 & 43,2 & 33,4 \\
\hline
\end{tabular}

Fuente: elaboración propia.

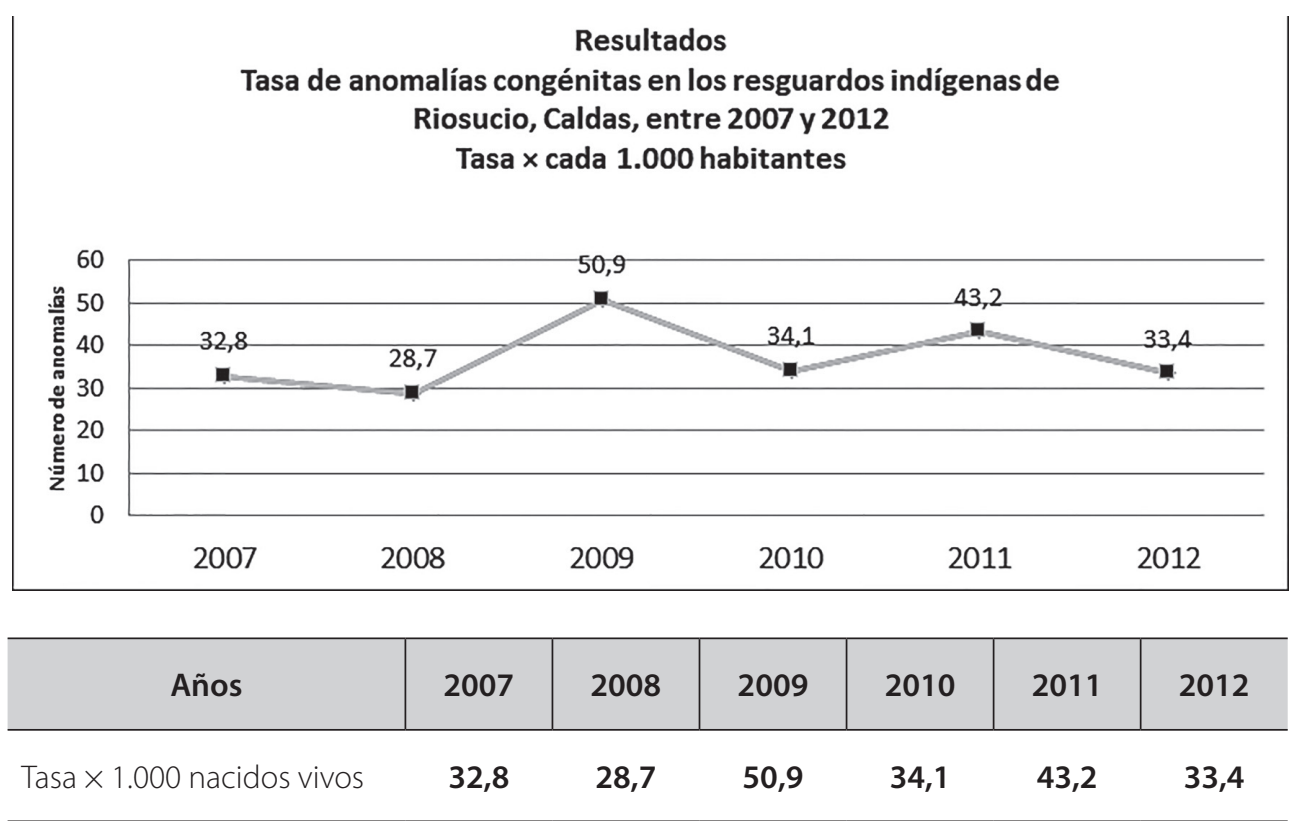

Gráfico 1. Tasa de anomalías congénitas por año, Riosucio, Caldas, Colombia, de 2007 a 2012

Fuente: elaboración propia.

El rango de edad de las madres de los pacientes con malformaciones congénitas fue de 12 a 42 años. La relación entre la edad de la madre del paciente y el diagnóstico principal dio una significancia estadística $(p<0,05)$. La edad de la madre en el momento del parto dio un promedio de 25,3 años (desviación estándar $=7,3$ ), y una media de 23 años de edad.
El rango de edad gestacional fue de 27 a 42 semanas. El tiempo promedio de edad gestacional fue de 38,4 semanas (desviación estándar $=1,9$ ) y media de 39 semanas. Hubo un $10 \%$ de casos prematuros, pero no se encontraron diferencias estadísticas significantes entre prematurez y la prevalencia de malformaciones congénitas. 
Se hallaron diferencias estadísticas significativas en la edad gestacional. El $87,2 \%$ de los casos con anomalías congénitas tuvieron un tiempo normal de embarazo y se identificaron diferencias estadísticas significantes entre la edad gestacional, específicamente la mayor a 37 semanas y el diagnóstico clasificado por sistemas anatomofisiológicos, como la displasia de cadera y la criptorquidia $(\mathrm{p}<0,05)$.

La edad de los pacientes al momento del diagnóstico tuvo un promedio de 13 meses (desviación estándar $=14,4)$ y la media fue de 9 meses. En ellos se encontró que las anomalías congénitas más frecuentes fueron: 1) Displasia de cadera $(32 \%), 2)$ Criptorquidia $(11,2 \%), 3)$ Malformaciones cardiovasculares (11,2\%), 4) Pie equino $(5,6 \%)$ e 5 ) Hidrocefalia $(3,2 \%)$. Otras cardiopatías congénitas se presentaron como el segundo diagnóstico más frecuente $(22,2 \%)$. Un segundo diagnóstico se presentó en el 21,6\% de los pacientes y un $9 \%$ de los pacientes presentaban un tercer diagnóstico.

En la displasia de cadera el $62,5 \%$ de las pacientes eran de sexo femenino $y$ el $37,5 \%$ de sexo masculino, lo cual evidencia que esta es la anomalía congénita más común en ambos sexos. El rango de edad de la madre más frecuente en este tipo de malformación fue entre los $16 \mathrm{y}$ los 20 años de edad.

Hay diferencias estadísticas significativas entre la edad del diagnóstico de la paciente y el resguardo indígena en donde los pacientes viven $(\mathrm{p}<0,05)$. En el resguardo indígena llamado San Lorenzo, el $71 \%$ de los casos fueron diagnosticados en el primer año de vida, en el resguardo de Escopetera-Pirsa el $65 \%$ fueron diagnosticados en el primer año de vida y en los otros resguardos, entre el 53 y el $57 \%$ de los casos fueron diagnosticados en el primer año.

Tabla 2. Variables con diferencias estadísticamente diferenciables $\left(\mathrm{Chi}^{2}: \mathrm{p}<0,05\right)$.

\begin{tabular}{|c|c|c|c|}
\hline \multicolumn{2}{|c|}{ Nombre de las variables } & \multirow{2}{*}{$\begin{array}{c}\text { Ji cuadrado } \\
0,016\end{array}$} & \multirow{2}{*}{$\begin{array}{c}\text { Observaciones } \\
59 \% \text { de los siete principales } \\
\text { diagnósticos }\end{array}$} \\
\hline Edad de la madre & $\begin{array}{l}\text { Diagnóstico } \\
\text { principal }\end{array}$ & & \\
\hline Edad gestacional & $\begin{array}{l}\text { Diagnóstico por } \\
\text { sistemas }\end{array}$ & 0,000 & $92,8 \%$ entre las $36-40$ semanas \\
\hline $\begin{array}{l}\text { Edad de diagnóstico } \\
\text { del paciente }\end{array}$ & Resguardo & 0,015 & $\begin{array}{l}87,2 \% \text { de los casos tuvieron } \\
\text { tiempo normal de embarazo }\end{array}$ \\
\hline $\begin{array}{l}\text { Edad de primera } \\
\text { consulta médica }\end{array}$ & $\begin{array}{l}\text { Diagnóstico } \\
\text { Principal }\end{array}$ & 0,038 & $\begin{array}{l}\text { 77,6\% tienen su primera consulta } \\
\text { antes del primer año de vida }\end{array}$ \\
\hline $\begin{array}{l}\text { Resguardo donde } \\
\text { vive el paciente }\end{array}$ & Tipo de zona & 0,024 & $\begin{array}{c}\text { La Montaña } 44 \% \\
\text { Cañamomo-Lomaprieta 22,4\% } \\
\text { San Lorenzo } 22,4 \%\end{array}$ \\
\hline Resguardo indígena & $\begin{array}{l}\text { Comunidades } \\
\text { procedentes }\end{array}$ & 0,000 & $\begin{array}{l}\text { Hay concentración de casos en el } \\
\text { 7\% de las veredas }\end{array}$ \\
\hline
\end{tabular}

Fuente: elaboración propia. 
Entre la edad de la primera consulta médica y el diagnóstico principal se encontró una diferencia estadística significativa $(\mathrm{p}<0,05)$. Se presentaron malfor- maciones congénitas más frecuentes en el sistema osteo-muscular $(50,4 \%)$, pero no hay una diferencia estadísticamente significativa al momento del diagnóstico.

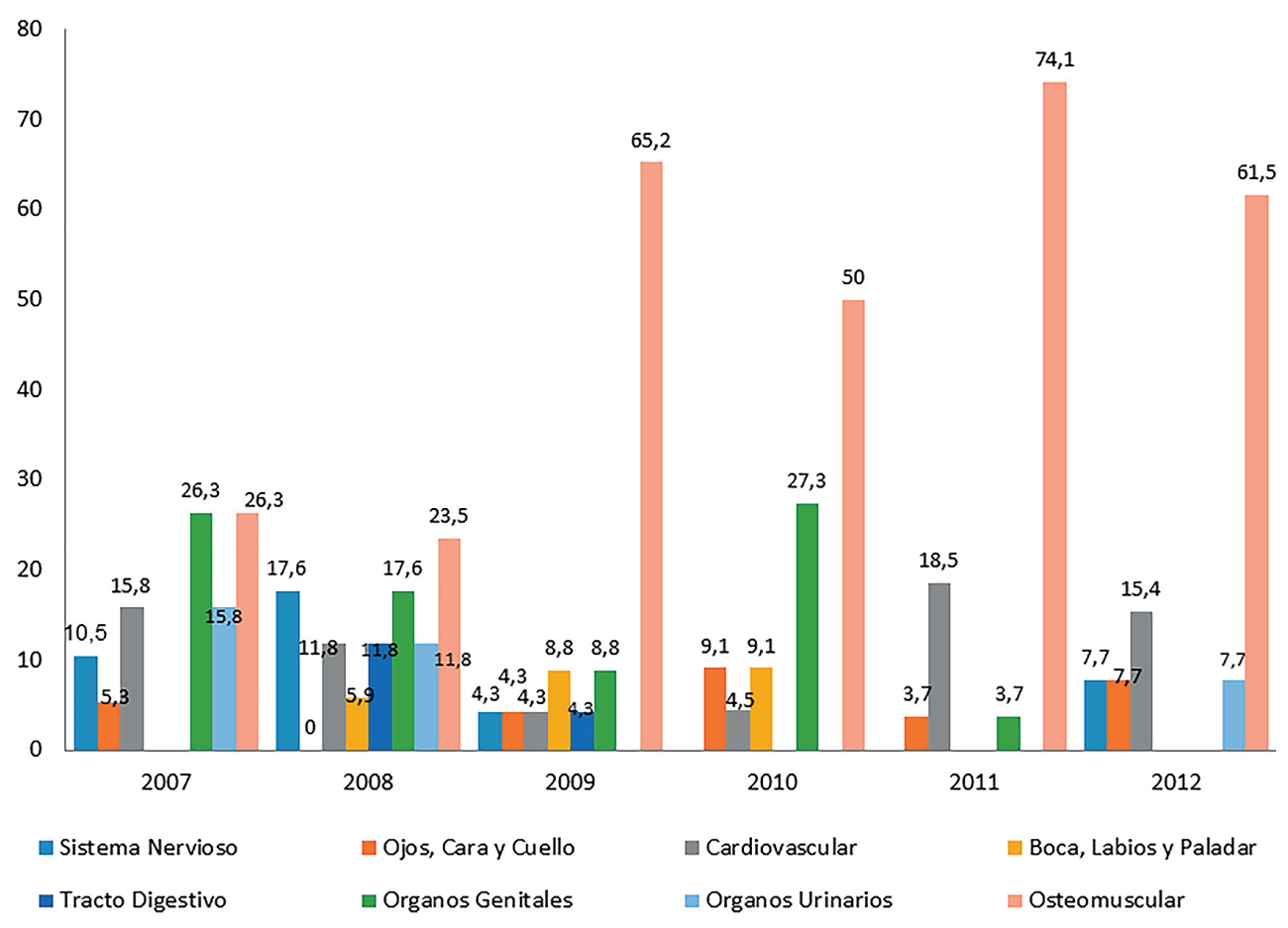

Gráfico 2. Distribución de anomalías congénitas, organizadas por frecuencia, por año, en los resguardos indígenas de Riosucio, Caldas; Población 61535

Fuente: Geoportal Dane, 2015.

Se encontraron diferencias estadísticamente significativas entre los resguardos donde los paciente viven y el tipo de zona donde viven $(p<0,05)$. La mayor parte de la población con malformaciones congénitas que viven en resguardos indígenas habitan en zonas rurales (88\%) y el resguardo de La Montaña tie- ne el mayor número de casos en la zona urbana $(21 \%)$. Se hallaron diferencias estadísticamente significativas $(\mathrm{p}<0,05)$ entre el resguardo indígena y el grupo comunitario donde viven los pacientes, $44 \%$ de los pacientes identificados procedían de comunidades del resguardo de La Montaña (gráfico 3). 


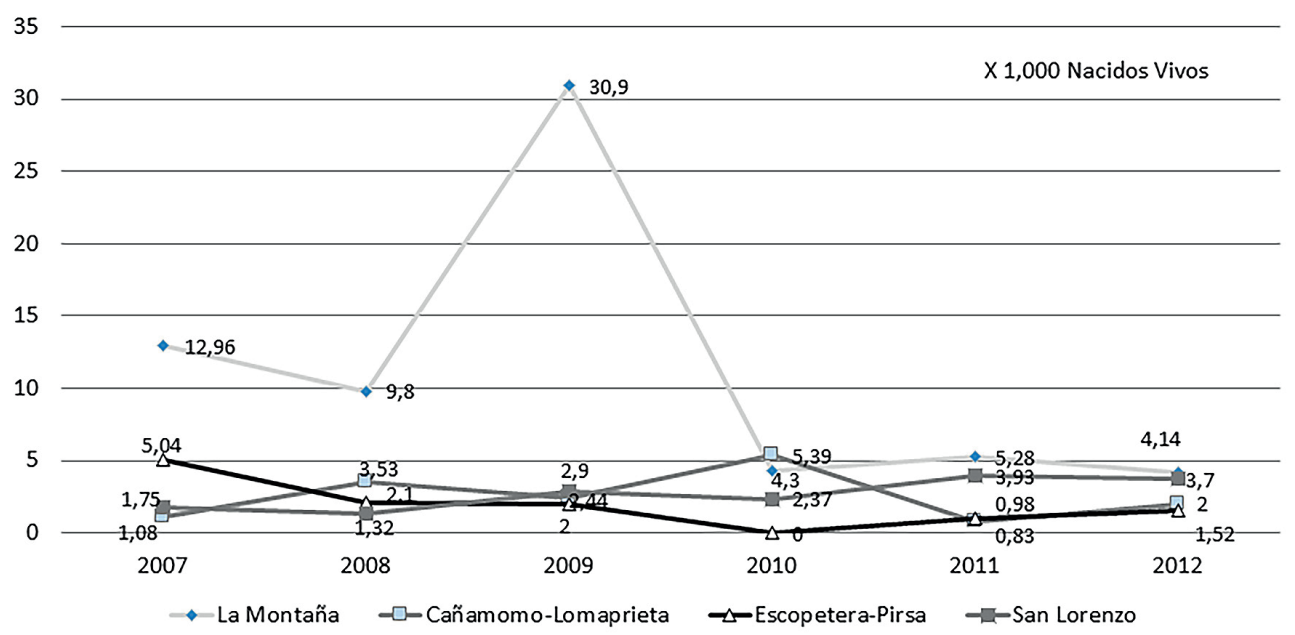

Gráfico 3. Tasa de anomalías congénitas en los resguardos indígenas de Riosucio, Caldas, Colombia entre 2007 y 2013

Fuente: elaboración propia.

Se encontraron diferencias estadísticamente significativas entre el grupo comunitario donde el paciente vive y el diagnóstico principal $(\mathrm{p}<0,05)$. Hay un mayor número de casos en los grupos comunitarios o veredas llamados Blandón, Lomitas (resguardo de San Lorenzo); Bonafont (resguardo de Escopetera-Pirsa); El Nevado y La Floresta (resguardo de La Montaña); y Sipirra y Tumbabarreto (resguardo de Cañamomo-Lomaprieta). Hay diferencias estadísticamente significativas entre el grupo comunitario o vereda donde viven los pacientes y el diagnóstico agrupado por sistemas $(\mathrm{p}<0,05)$.

El gráfico 4 es una cartografía social que muestra la distribución geográfica en las veredas de cada uno de los cuatro resguardos indígenas del municipio de Riosucio, de acuerdo al número de casos y al sistema afectado según la anomalía encontrada. Es así como se observa una concentración de casos en la zona urbana con más casos de malformaciones de los sistemas osteomusculares y genitourinarios. En veredas cercanas a la zona urbana, en los resguardos de San Lorenzo y La Montaña, se observan más malformaciones osteomusculares y cardiovasculares. Existe en el resguardo de Escopetera-Pirsa, en la zona rural de Bonafont y San Antonio, una concentración de casos diferentes. A nivel general del municipio, en las zonas rurales los casos de malformaciones no son concentrados por número, por tipo de sistema, ni por vereda y se observan en la cartografía más extendidos en las diferentes veredas de Riosucio. 


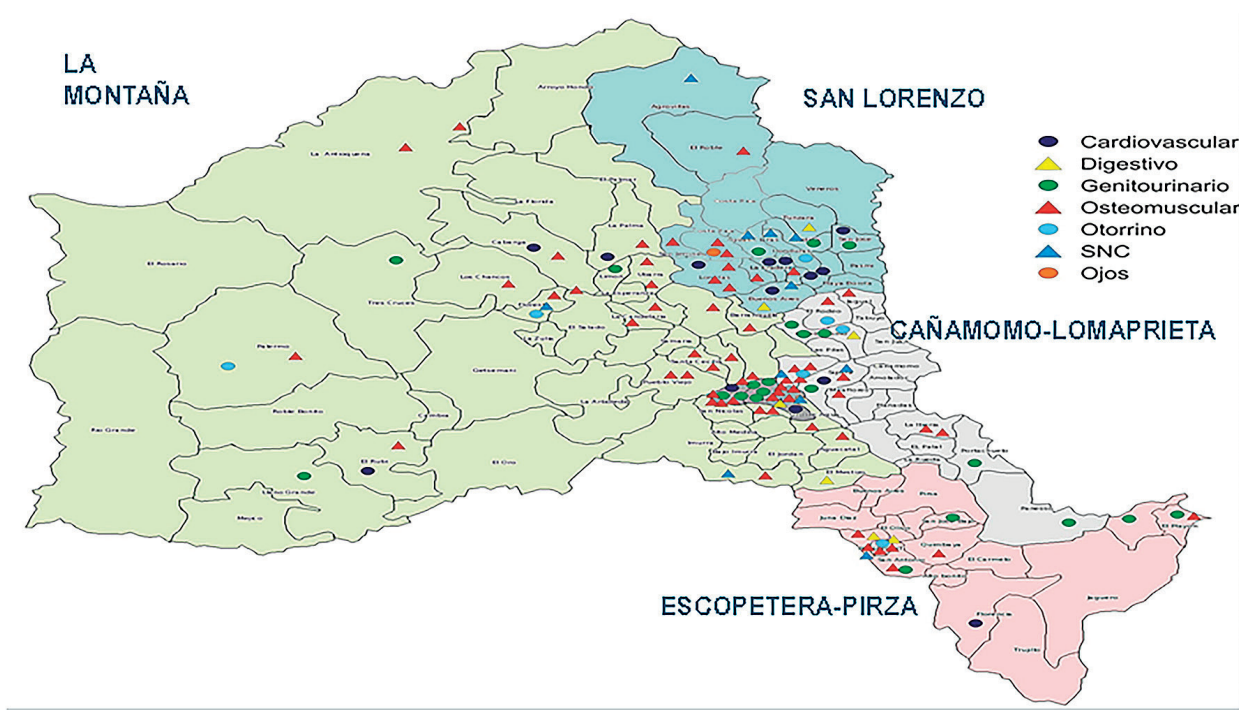

Gráfico 4. Cartografía social de anomalías congénitas en los resguardos indígenas de Riosucio, Caldas, Colombia

Fuente: Adaptado de Wikimedia Commons (mapa de Riosucio, Caldas). Las convenciones son del autor. Disponible en https://commons.wikimedia.org/w/index.php? search=mapa+de+riosucio\%2C+caldas\&title $=$ Special\%3ASearch \&go $=$ Go\&ns0 $=1 \&$ ns $6=1 \&$ ns $12=1 \&$ ns $14=1 \&$ ns $100=1 \& \mathrm{~ns} 106=1 \# / \mathrm{media} /$ File:Colombia_-_Caldas_-_Riosucio.svg

\section{Discusión}

En Colombia, desde 1994, las anomalías congénitas se han reportado como la segunda causa de mortalidad en los niños menores de cinco años y a nivel mundial esta se ha mantenido entre las cinco primeras (35-37). La proporción de malformaciones que se ha reportado en los Estados Unidos de América es de 3,12\% (28) y se encontró en esta investigación una proporción ligeramente más alta, $3,7 \%$. De los resultados en este estudio, la tasa de anomalías congénitas dio un promedio por año de $36,97 \times$ cada 1.000 nacimientos, lo que es mayor a resultados de otras ciudades del país, como Cartagena (32 $\times 1.000$ nacimientos $)$, Cali $(23 \times 1.000$ nacimientos $)$, Barranquilla $(22 \times 1.000$ nacimientos $)$ y Bogotá (de 27 a $30 \times 1.000$ nacimientos) $(11,38$, 39). Esto puede sugerir el predominio de la población étnica homogénea que mantiene el municipio de Riosucio y la concentración de esta población dentro de sus resguardos.

Se observó una diferencia de una investigación del Centers for Desease Control and Prevention en Nueva York $(6,40)$, donde se citó una heterogeneidad entre las malformaciones congénitas y el género del paciente. En esta investigación, por el contrario, se encuentra una homogeneidad entre las malformaciones congénitas y el género (36, 40-42).

La edad de la madre presentó un promedio de 25,3 años, similar a otras investigaciones colombianas $(11,14,41,42)$. La edad gestacional tuvo un promedio de 
38,4 semanas, similar a los resultados de otras investigaciones $(36,43)$. La edad de los pacientes con malformaciones congénitas al momento del diagnóstico dio un promedio de 13 meses, similar a otro estudio en el mismo departamento de Caldas, Colombia, que reportó 15 meses (42).

Las anomalías más frecuentes en esta investigación fueron la displasia de cadera, la criptorquidia, las cardiopatías, el pie equino varo y equino valgo y la hidrocefalia. Resultados similares se encontraron en estudios previos en Manizales (42), Universidad Javeriana (41) y Universidad del Valle (43).

Aunque el estrato social no fue una de las variables de este estudio sobre anomalías congénitas en Riosucio, el Observatorio de Salud del departamento de Caldas indica que el 74,82\% de la población en Riosucio está clasificada en los estratos 1 y 2. Los resultados de una tasa mayor de lo normal en las poblaciones indígenas encontradas aquí puede sugerir una asociación entre este evento y la situación económica, como se ha demostrado en otras investigaciones realizadas en otras zonas de Colombia (43-46).

La mayor parte de los registros de la población con malformaciones anómalas estudiadas se encuentra en la zona rural del municipio ( $88 \%$ ). La mayor parte de los pacientes identificados (44\%) viven en las veredas del resguardo indígena de La Montaña.

Desde el inicio hasta el final de la inves56 tigación, que tuvo una duración de un año, hubo dificultades principalmente para acceder a la información por parte de las entidades donde fueron atendidos los pacientes, a pesar de seguir los trámites solicitados. A pesar de haber un buen número de casos, el hecho de no contar con todas las variables requeridas excluyó una gran cantidad de las historias clínicas disponibles. El instrumento identificado como "Anomalías Congénitas Cód. INS:735” del Instituto Nacional de Salud exigía demasiadas variables que no se encontraron en las historias clínicas de la muestra, por lo cual fue necesario crear un instrumento propio para esta investigación con las variables específicas disponibles de las muestras.

\section{Conclusiones}

Se encontró una tasa de anomalías congénitas en la población indígena de los Embera-Chamí de Riosucio, Caldas, mayor que el promedio de la población en Colombia. De otro lado se halló una tasa de malformaciones congénitas similar entre los resguardos estudiados en Riosucio, excepto en el resguardo indígena de La Montaña, el cual fue mayor en el año 2009. La mayor parte de anomalías congénitas se presentaron como malformaciones en el sistema osteo-muscular.

Se identifica una importante diferencia entre los indicadores de anomalías congénitas en la población indígena con respecto a la población general, lo que sugiere que por las características étnicas, históricas, geográficas y socio-culturales propias de ellos, se deben realizar estudios con indicadores propios de la población indígena Embera para aplicar un mejor diagnóstico, tratamiento $y$ control. 


\section{Sugerencias}

Se sugiere organizar un programa de identificación y seguimiento de enfermedades congénitas en la población indígena Embera-Chamí del municipio de Riosucio, Caldas, Colombia, para facilitar investigaciones de control a mediano y largo plazo de estas patologías. También se deben de iniciar estudios más específicos de malformaciones congénitas, enfocándose en el resguardo indígena de La Montaña, del municipio de Riosucio, Caldas, donde se presentaron más casos.
Orientar estudios de malformaciones congénitas de la población Embera-Chamí del municipio de Riosucio, Caldas, Colombia, hacia los factores de riesgo, que el estudio sugiere que influyen en el desencadenamiento de este tipo de patologías.

\section{Agradecimientos}

Agradecimientos al CDC, Tephinet y FETP por el apoyo financiero a la investigación. A las instituciones hospitalarias por el acceso a las historias clínicas. 


\section{Referencias bibliográficas}

1. DANE. Censo General 2005: Perfil Riosucio, Caldas [Sitio en internet]. 2005 [citado 2013 febr. 10]. Disponible en: http://www.dane.gov.co/files/censo2005/ PERFIL_PDF_CG2005/17614T7T000. PDF

2. DANE. Proyecciones de Población departamentales y municipales. [Sitio en internet]. 2005 [citado 2013 febr.20]. Disponible en: .www.dane.gov.co/files/ investigaciones/...20/ProyeccionMunicipios2005_2020.xls?

3. Comisión Nacional de Trabajo y Concertación de la Educación para los Pueblos Indígenas, Contcepi. Perfil del sistema educativo indígena propio -S.E.I.P-. [Internet]. 2013 [citado 2013 febr. 20]. Disponible en: http://www.ut.edu.co/administrativos/images/DOCUMENTOS $\% 20$ ADMINISTRATIV OS/ASOCIACIONES/ CABILDO_INDIGENA/seip.pdf

4. Cepal. Pueblos indígenas y afrodescendientes de América Latina y el Caribe: Información sociodemográfica para políticas y programas. [Sitio en internet]. 2013 [citado 2013 febr. 20]. Disponible en: http://www.eclac.cl/publicaciones/ xml/0/25730/pueblosindigenas_final-web. pdf\#page $=135$

5. Observatorio ADPI. Observatorio por la autonomía y los derechos de los pueblos indígenas en Colombia. [Sitio en internet]. [fecha desconocida] [citado 2013 mzo. 12]. Disponible en: http://observatorioadpi.org/emberach

6. ONIC. Autoridad nacional de Gobierno indígena. [Sitio en internet]. [fecha desconocida] [citado 2012 nov. 14]. Disponible en: http://cms.onic.org.co/

7. Marrugo N. Multiculturalismo, derechos humanos y derechos de los indígenas en Colombia: Práctica de la ablación en las

58 niñas de la tribu Embera Chamí. Justicia Juris. 2014;10(1):32-42.
8. Sabogal J. Embera Wera: vida, poder y resistencia. Ciudad Paz-Ando. 2014;7(1):198-214.

9. Siagama L. El pensamiento Embera Chamí: un análisis filosófico. [Internet]. 2017 [citado 2013 febr. 21]. Disponible en: http://repository.unad.edu.co/bitstream/10596/13777/1/18603331.pdf

10. Corte Constitucional de Colombia. Sentencia T-601/11 [Sitio en internet]. 1986, jun. [citado 2013 mzo. 12]. Disponible en: http://www.corteconstitucional.gov. co/relatoria/2011/T-601-11.htm

11. Vasco L. Algunas notas sobre historia Chami. [Sitio en internet]. Ponencia para el Seminario Regional sobre Historia Indígena, Secretaría de Desarrollo de Antioquia, Medellín. 1986 [citado 2012 oct. 31]. Disponible en http://www.luguiva.net/ admin/pdfs/ALGUNAS $\% 20$ NOTAS $\% 20$ SOBRE\%20HISTORIA\%20CHAMI.pdf

12. Gobierno Nacional. Informe del Gobierno Nacional a las Comisiones Primeras del Congreso de la República. Avances en la Ejecución de la Ley 1448 de 2011. [Internet]. 2013 [citado 2013 febr. 28]. Disponible en: http://www.acnur.org/ t3/uploads/media/2882_COI_Colombia_ Informe_Comisione s_Primeras_Congreso_2014.pdf?view=1

13. Cridec. Situación del derecho a la salud de los pueblos indígenas Emberá-Chami del municipio de Riosucio, Caldas. [Sitio en Internet]. 2017 [citado 2012 ag. 10]. Disponible en: http://www.observatorio.saluddecaldas.gov.co/desca/articulos/Contexto $\% 20$ general $\% 20 \mathrm{del} \% 20$ pueblo\%20embera_2017.pdf

14. Rondón F, Osorio JC, Peña AV, Garcés HA, Barreto $G$. Diversidad genética en poblaciones humanas de dos regiones colombianas. [Sitio en internet]. 2008 [citado 2012 ag. 30] Disponible en: http:// www.bioline.org.br/request?rc08042 
15. Colombialink. Los pueblos indígenas colombianos al momento de la conquista. [Sitio en internet]. 2012 [citado 2012 oct. 01]. Disponible en: http://www.colombiamania.com/historia/index_historia/02 la_conquista/0_0003_indigenas_epoca_ conquista.html

16. Sabogal J. Experiencias educativas y construcciones del Yo en comunidades Embera: Una mirada biográfica. [Sitio en internet]. 2014 [citado 2012 oct. 01]. Disponible en: https://www.researchgate.net/ publication/276291220

17. Cardona JA, Rivera Y, Carmona J. Expresión de la interculturalidad en salud en un pueblo Emberá-Chamí de Colombia. Rev Cubana Salud Pública. 2015;41(1):77-93.

18. Melo MA. Parentesco y familia en algunas comunidades indígenas colombianas: persistencia y cambio. Av. Enferm, [Internet].1995. [citado 2018 mzo. 18];13(7). Disponible en: https://revistas. unal.edu.co/index.php/avenferm/article/ view/16473

19. Cardona JA. Sistema médico tradicional de comunidades indígenas Emberá-Chamí del Departamento de Caldas-Colombia. Rev de Salud Pública. [Internet]. 2012 [citado 2018 mzo. 18];14(4). Disponible en: https://revistas. unal.edu.co/index.php/revsaludpublica/ article/article/view/23329

20. Cardona JA, Marín D, Salazar R. Estudio ecológico sobre parasitismo intestinal, anemia y estado nutricional en indígenas Emberá-Chamí. Medicina \& Laboratorio. 2013;19:381-394.

21. Unidad para la Atención y Reparación Integral a las Víctimas, UARIV. Quinto informe trimestral al Auto 052 de 2013 mediante el cual la Corte Constitucional solicitó información sobre las medidas adoptadas para atender a las comunidades 44 Intervención Institucional en la comunidad Embera Chami Embera Katío (Chocó) y Embera Chamí (Risa- ralda) que se encuentran desplazadas en la ciudad de Bogotá, en el marco de lo dispuesto por la sentencia T-025 de 2004 y el Auto 004 de 2009. Documento virtual inédito. [Internet]. 2014a. [citado 2014 Febr. 28]. Disponible en: https://www.corteconstitucional.gov. co/T-025-04/AUTOS\%202012/254.\%20 Auto\%20del\%209Auto\%20informaci\%C3\%B3n\%20Embera.pdf

22. Dirección Local de Salud del Municipio de Riosucio, Caldas. Perfil Epidemiológico. Edición única. Riosucio, Caldas: Alcaldía Municipal; 2011.

23. Zarante I. Implementación, desarrollo y evaluación de un programa de vigilancia en salud pública y seguimiento de pacientes con malformaciones congénitas en la ciudad de Bogotá D.C., Colombia, basado en la metodología de Estudio Colaborativo Latinoamericano de Malformaciones Congénitas (ECLAMC). [Tesis doctoral]. [Bogotá D.C.]: Instituto de Genética Humana; 2011.

24. Bernal J, Zarante I. Malformaciones y anomalías congénitas: impacto y futuro. Biomedica [Internet]. 2009 [citado 2018 mzo. 19];29(1):7-8. Disponible en: https:// www.revistabiomedica.org/index.php/ biomedica/article/view/34

25. González N. Protocolo de vigilancia en salud pública: Defectos congénitos [Internet]. 2015 mzo. 19 [revisado 2013 abr. 4; citado 2015 nov. 4]. Disponible en: https:// cruevalle.org/files/PRO-Derectos-Congenitos.pdf

26. OMS. Clasificación internacional de las enfermedades, ICD-10. [Sitio en internet]. 2010 [citado 2013 abr. 04]. Disponible en: http://www.iqb.es/patologia/ e15_009.htm

27. Instituto Nacional de Salud. Informe final de anomalías congénitas año 2011. [Sitio en internet]. 2011 [citado 2013 abr. 04] Disponible en: http://www.ins.gov.co/ lineas-de-accion/Subdireccion-igilancia/ Informe\%20de\%20Evento\%20Epide- 
miolgico/Anomalias\%20cong\%C3\%A9nitas\%202011.pdf

28. Sistema Nacional de Información ¿Qué es la georreferenciación? [Sitio en internet]. 2014 [citado 2013 mzo. 04]. Disponible en: http://infdigital.sni.gob. ec/?p=1007

29. Carreño MC, González V. De la pobreza a la indigencia: Asentamiento indígena Embera Chamí en Caldas, Colombia. Bogotá D.C. Diálogo de Saberes. 2014;41:159-70.

30. Wieczorek J, Bloom D, Constable H, Fang J, Koo M, Spencer C, et al. Georeferencing quick reference guide. [Sitio en internet]. 2012 oct. 08 [citado 2013 abr. 10]. Disponible en: http://www.herpnet. org/herpnet/documents/GeoreferencingQuickGuide.pdf

31. Instituto de Evaluación Tecnológica en Salud. Guía de práctica clínica: Detección e anomalías congénitas en el recién nacido. [Sitio en internet]. Edición única. Bogotá D.C.: Ministerio de Salud y Protección Social-Colciencias; 2013 [revisado 2014 nov.; citado 2016, junio]. Disponible en: https://www.minsalud.gov.co/sites/rid/ Lists/BibliotecaDigital/RIDE/INEC/IETS/ GPC_Completa_Anom_Conge.pdf

32. World Health Organization. International statistical classification of diseases and related health problems. [Sitio en internet]. vol, 2, 5ta ed. Geneva, Switzerland: World Health Organization; 2016. [revisión 2016 nov; citado 2017 en.]. Disponible en: https://www.who.int/classifications/icd/ICD10Volume2_en_2010.pdf

33. Restrepo GA. Diagnóstico prenatal de anomalías congénitas: ¿se cumple esta política en Colombia? Rev CES Med. 2018;32(3):226-34.

34. Ministerio de Salud, República de Colombia. Resolución № 008430 de 1993 (4 de octubre de 1993). [Sitio en internet]. http://www.urosario.edu.co/urosario files/a2/a24fb07a-f561-4fcc-b611-affff4374bb7.pdf

35. Google Maps. Mapas satelitales de rutas y planos de calles de Riosucio. Coordenadas GPS y Geooglemaps de Caldas, Colombia. [Sitio en internet]. 2009 [citado 2013 mzo. 01]. Disponible en: http://www. demapasyrutas.com/Caldas/Riosucio/

36. Nazer $\mathrm{H}$, Cifuentes O. Prevalencia al nacimiento de malformaciones congénitas en las maternidades chilenas participantes en el ECLAMC en el período 20012010. Rev méd. Chile [Internet]. 2014 sept. [citado 2016 mzo. 20];142(9):11506. Disponible en: https://scielo.conicyt. $\mathrm{cl} /$ scielo.php?script=sci_arttext\&pi$d=$ S0034-98872014000900009\&Ing

37. Castellanos J, Forero J. Incidencia de malformaciones congénitas presentes en recién nacido en el Hospital Mario Gaitán Yanguas-Soacha, durante el periodo del año 2010-2014. [Tesis]. Bogotá D.C.: Universidad de Ciencias Aplicadas y Ambientales-U.D.C.A.; 2015.

38. Secretaría de Gobierno de Bogotá. Los Embera desplazados en Bogotá. [Internet]. 2014. [citado 2016 Mzo. 20]. Disponible en: http://wwwold.gobiernobogota.gov.co/descargas/Cartilla_de_Lineamientos_Embera.pdf

39. La Mochila Ambulante. Lo que mal empieza... Agencia Prensa Rural. [Internet] 2014 febr. 11. [citado 2016 Mzo. 20]. Disponible en: http://prensarural.org/spip/ spip.php?article13336

40. WHO. Congenital anomalies. [Sitio en internet]. 2012 [citado 2013 abr. 01]. Disponible en: http://www.who.int/ mediacentre/factsheets/fs370/en/

41. WHO/CDC/ICBDSR. Birth defects surveillance: a manual for programme managers. Geneva: World Health Organization; 2014. 
42. Zarante I, Franco L, López C, Fernández $\mathrm{N}$. Frecuencia de malformaciones congénitas: evaluación y pronóstico de 52.744 nacimientos en tres ciudades colombianas. Biomédica. 2010;30(1):65-71.

43. Acosta J, Álvarez C, Castaño J, Gaitán L, León A, Mariño I, et. al. Prevalencia de las cardiopatías congénitas en un hospital de la ciudad de Manizales, Colombia, años 2004 y 2008. Arch de Medi. 2009;9(2):99-109.

44. Cardona J, Llanes O. Hipertensión arterial y sus factores de riesgo en indígenas Embera-Chamí. Rev CES Med. 2013;27(1):31-4.
45. Monsalve AM, Londoño IC, Ocampo J, Cruzi DF, Saldarriaga W, Isaza C. Distribución geográfica en Cali, Colombia de malformaciones congénitas, Hospital Universitario del Valle, marzo de 2004-febrero de 2005. Colomb Med. 2007;38(1):47-51.

46. Alcaldía Municipal de Riosucio. Perfil Epidemiológico de Riosucio, Caldas. [Sitio en internet]. Edición única. Alcaldía Municipal: Riosucio, Caldas. [Internet] 2014 [citado 2015 abr. 18]. Disponible en: http:// www.observatorio.saluddecaldas.gov.co/ desca/perfil/Perfil_epidemiol\%C3\%B3gico_Riosucio_2013.pdf 
INVESTIGACIONES ANDINA No. 39, Vol. 21 